\title{
Human rhinoviruses enter and induce proliferation of $B$ lymphocytes
}

DOI:

10.1111/all.12931

\section{Document Version}

Accepted author manuscript

Link to publication record in Manchester Research Explorer

\section{Citation for published version (APA):}

Aab, A., Wirz, O., van de Veen, W., Söllner, S., Stanic, B., Rückert, B., Aniscenko, J., Edwards, M. R., Johnston, S. L., Papadopoulos, N., Rebane, A., Akdis, C. A., \& Akdis, M. (2017). Human rhinoviruses enter and induce proliferation of B lymphocytes. Allergy: European Journal of Allergy and Clinical Immunology, 72(2), 232-243. https://doi.org/10.1111/all.12931

\section{Published in:}

Allergy: European Journal of Allergy and Clinical Immunology

\section{Citing this paper}

Please note that where the full-text provided on Manchester Research Explorer is the Author Accepted Manuscript or Proof version this may differ from the final Published version. If citing, it is advised that you check and use the publisher's definitive version.

\section{General rights}

Copyright and moral rights for the publications made accessible in the Research Explorer are retained by the authors and/or other copyright owners and it is a condition of accessing publications that users recognise and abide by the legal requirements associated with these rights.

\section{Takedown policy}

If you believe that this document breaches copyright please refer to the University of Manchester's Takedown Procedures [http://man.ac.uk/04Y6Bo] or contact uml.scholarlycommunications@manchester.ac.uk providing relevant details, so we can investigate your claim.

\section{OPEN ACCESS}




\section{B Lymphocytes}

$1 \quad$ Alar Aab ${ }^{1,2^{*}}$, Oliver Wirz ${ }^{2 *}$, Willem van de Veen ${ }^{2}$, Stefan Söllner ${ }^{2}$, Barbara Stanic $^{2}$, Beate Rückert $^{2}$, Julia $^{2}$

2 Aniscenko ${ }^{3,4}$, Michael R. Edwards ${ }^{3,4}$, Sebastian L. Johnston ${ }^{3,4}$, Nikolaos G. Papadopoulos ${ }^{5,6}$, Ana

3 Rebane ${ }^{1}$, Cezmi A. Akdis ${ }^{2}$, and Mübeccel Akdis ${ }^{2}$

$4{ }^{1}$ Institute of Biomedicine and Translational Medicine, University of Tartu, Estonia;

$5 \quad{ }^{2}$ Swiss Institute of Allergy and Asthma Research (SIAF), University of Zürich, Davos, Switzerland;

$6{ }^{3}$ Airway Disease Infection Section, National Heart and Lung Institute, Imperial College London,

7 London, United Kingdom;

$8 \quad{ }^{4} \mathrm{MRC} \&$ Asthma UK Centre for Allergic Mechanisms of Asthma, London, United Kingdom.

$9 \quad{ }^{5}$ Allergy Department, 2nd Pediatric Clinic, University of Athens, Athens, Greece.

$10{ }^{6}$ Centre for Pediatrics \& Child Health, Institute of Human Development, The University of Manchester, 11 United Kingdom.

* These authors contributed equally to this work.

Correspondence to: Mübeccel Akdis, Swiss Institute of Allergy and Asthma Research (SIAF), Obere 


\section{Abstract}

Background: Human rhinoviruses (HRV) are one of the main causes of virus induced asthma exacerbations. Infiltration of B lymphocytes into the subepithelial tissue of the lungs has been demonstrated during rhinovirus infection in allergic individuals. However, the mechanisms through which HRVs modulate the immune responses of monocytes and lymphocytes are not yet well described.

Objective: To study the dynamics of virus uptake by monocytes and lymphocytes, and the ability of HRVs to induce activation of in vitro cultured human peripheral blood mononuclear cells.

Methods: Flow cytometry was used for the enumeration and characterization of lymphocytes. Proliferation was estimated using ${ }^{3} \mathrm{H}$-thymidine or CFSE labelling and ICAM-1 blocking. We used bead based multiplex assays and quantitative PCR for cytokine quantification. HRV accumulation and replication inside B lymphocytes was detected by a combination of in situ hybridation (ISH), immunofluorescence and with PCR for positive strand and negative strand viral RNA. Cell images were acquired with imaging flow cytometry.

Results: By means of imaging flow cytometry, we demonstrate a strong and quick binding of HRV types 16 and $1 \mathrm{~B}$ to monocytes, and slower interaction of these HRVs with CD4+ T cells, CD8+ T cells and CD19+ B cells. Importantly, we show that HRVs induce the proliferation of B cells while addition of anti-ICAM-1-antibody partially reduces this proliferation for HRV16. We prove with ISH that HRVs can enter B cells, form their viral replication centers and the newly formed virions are able to infect HeLa cells. In addition, we demonstrate that similarly to epithelial cells, HRVs induce the production of pro-inflammatory cytokines in PBMCs.

Conclusion: Our results demonstrate for the first time that HRVs enter and form viral replication centers in B lymphocytes and induce the proliferation of B cells. Newly formed virions have the 
41 capacity to infect other cells (HeLa). These findings indicate that the regulation of human rhinovirus

42 induced B cell responses could be a novel approach to develop therapeutics to treat the virus-

43 induced exacerbation of asthma.

44 Keywords: Asthma; B lymphocyte; Human rhinovirus.

\section{Abbreviations}

CFSE carboxy-fluorescein succinimidyl ester

47

DAPI 4', 6-diamidino-2-phenylindole

$48 \quad H R V$

human rhinovirus

49 ICAM-1

intercellular Adhesion Molecule 1 also known as CD54

$50 \quad$ IFN

interferon

$51 \quad$ IL

interleukin

$52 \quad$ LDLR

low-Density Lipoprotein Receptor

$53 \quad$ LRT

lower respiratory tract

$54 \mathrm{MOI}$

multiplicity of infection

$55 \quad$ PBMC

peripheral blood mononuclear cell

56 TCID

tissue culture infective dose

$57 \quad$ Th

T helper cell

$58 \quad$ URT

upper respiratory tract

59 UV HRV

ultraviolet inactivated HRV

60

VRNA

viral RNA

61 


\section{Introduction}

Virus-induced exacerbations are the major cause of morbidity and mortality among asthma patients. Human rhinoviruses (HRVs) are the most common triggering factors of acute asthma exacerbations $(1,2)$; however, evidence demonstrating a causal relationship between HRV infection and asthma exacerbation is mostly limited to epidemiological studies and the immunological mechanisms are poorly understood. T helper (Th) 2 cells are considered the main initiating effector cell type in atopic asthma in general, and asthma exacerbations in particular. However, exaggerated Th2 cell activities alone do not explain all aspects of asthma and exacerbations. In addition, they cannot explain susceptibility to viral infection, shared between asthma and chronic obstructive pulmonary disease (3).

HRV was the first identified member of the Picornaviridae family. The picornaviruses are nonenveloped viruses with a single stranded, positive sense RNA genome. The family consists of twelve genera, including the Enterovirus genus, to which HRV belongs (4). On nucleic acid identity this genus can be subdivided further into ten species, from which three are HRV-A,-B and -C. ICAM-1 (intercellular adhesion molecule 1, also known as CD54) is the virus entry receptor for the major group of rhinoviruses, such as HRV16 and HRV14 serotypes, and LDLR (low-density lipoprotein receptor) is the receptor for minor group rhinoviruses, such as HRV1B and HRV29 (5). It has been recently found that HRV-C enters the cells using Cadherin-related family member 3 (CDHR3) $(6,7)$. ICAM-1 and LDLR are expressed on the surface of many different cell types, including those that are active in respiratory immune responses, such as monocytes and airway macrophages, dendritic cells, airway eosinophils, mast cells, B lymphocytes and activated T lymphocytes $(8,9)$. Less is known about expression of CDHR3 but it is highly expressed in human lung tissue (10) and bronchial epithelium (7).

Among terminally differentiated bronchial epithelial cells, HRV replication has been documented predominantly in ciliated epithelial cells (11). The HRV-infected epithelial cells release a variety of 
inflammatory factors, which orchestrate proliferation, chemotaxis, and activation of immune cells, resulting in an amplification of inflammation. It is generally believed that HRVs are not able to infect white blood cells. In contrast, it has been recently shown that HRVs are able to infect human macrophages (12), T cells (13-15) and Ramos cells (human B lymphocyte cell-line) (16). Even then, and similar to bronchial epithelial cells, only a small fraction of cells are positive for virus replication (12). Innate activation of monocytes upon a viral infection(17), and close contact of upper and lower airway epithelium cells with infiltrating inflammatory leukocytes, granulocytes, dendritic cells and monocytes suggests that these cells might influence immune responses to HRV in vivo, and be important to explain processes during asthma exacerbations (18).

In addition to induction of asthma exacerbations, there is likely a causative link between HRV infections and the development of childhood asthma (19), but yet fundamental questions persist about mechanisms linking this common pathogen to the disease. As it is generally thought that asthma results from over-zealous responses to innocuous antigens or poor regulation of the immune system, the details of how HRV infection interacts with the host immune system could be the key in uncovering the underlying mechanism of the development of asthma. Understanding disease pathophysiology is important for the development of effective and evidence-based treatments that are currently a major unmet need for both asthma exacerbations and asthma onset.

In the present study, we investigated the ability of HRVs to bind and activate lymphocytes using in vitro cultured human peripheral blood mononuclear cells (PBMCs). We investigated the dynamics of infection in monocytes and lymphocytes and demonstrate for the first time that HRVs enter, form viral replication centers and induce strong proliferation of B cells. 


\section{Methods}

\section{Study subjects}

Sixteen subjects ( 13 female, 3 male) adult volunteers participated in the study. All subjects were healthy in the moment of drawing blood. The status of possible infections was followed three days before and three days after drawing the venous blood and confirmed not to be other than healthy.

\section{Generation of HRV stocks}

HRV serotypes 14, 16, 1B and 29 were propagated in Ohio HeLa cells using standard protocol (20). Virus stocks were titrated by infecting HeLa monolayers with serially diluted HRV and assessing cytopathic effect to estimate their $50 \%$ tissue culture infective dose (TCID50)/ml by Kremser method. The identities of all HRVs were confirmed by neutralization using serotype-specific antibodies (ATCC). TCID50/ml was expressed as multiplicity of infection (MOI) based on TCID50 $0.1 \mathrm{~mL}$ of a TCID50 virus of $1 \mathrm{e} 7$ per $\mathrm{mL}$ on $1 \mathrm{e} 6$ cells would be an $\mathrm{MOI}$ of 1 .

\section{Ultraviolet inactivation of virus and "Mock" solution}

UV-inactivated virus was prepared by irradiating the virus suspensions in a 24-well tissue culture dish on ice for 10 minutes with a $75 \mathrm{~W}$ UV source $(254 \mathrm{~nm})$ at a distance of $5 \mathrm{~cm}$. Treatment resulted in complete loss of infectious titer as estimated by titration assay. To produce "mock" solution, Ohio HeLa cells were cultured as for virus production without viruses to the culture.

\section{Isolation and culturing of PBMCs}

PBMCs were obtained from heparinized whole blood by Biocoll (Biochrom KG, Berlin, Germany) density gradient centrifugation. Cells were washed three times with PBS (0.02\% EDTA added) and suspended in RPMI 1640 medium supplemented L -glutamine ( $2 \mathrm{mmol} / \mathrm{L})$, MEM vitamin, penicillin 
$129(100 \mathrm{U} / \mathrm{mL})$, streptomycin $(100 \mu \mathrm{g} / \mathrm{mL})$, kanamycin, nonessential amino acids, sodium pyruvate (Life

130 Technologies, Carlsbad, Calif), and with $10 \%$ heat inactivated fetal calf serum (FCS; Invitrogen).

131 Further we call the RPMI 1640 medium together with supplements as CRPMI (complete RPMI).

132

133

134

135

136

137

138

139

140

141

142

143

144

145

\section{Culturing of PBMC with virus}

PBMC were exposed to HRV at $\mathrm{MOI}=1.0$ or 10 or other when indicated. As controls, UV-treated HRVs or no virus ("mock"), were used.

\section{Proliferation assay with ${ }^{3} \mathrm{H}$-thymidine}

After 5 day of stimulation with unlabelled active or UV inactivated or without viruses (mock) in triplicates, PBMCs were pulsed for 8 hours with ${ }^{3} \mathrm{H}$-thymidine, harvested with a Tomtec plate washer, and counted on a Betaplate counter. Results were expressed as a stimulation index (SI).

\section{CFSE labelling}

PBMC were washed in PBS twice, suspended in $10 \mathrm{~mL}$ PBS containing 5\% FBS. CSFE (from $10 \mathrm{mM}$ stock and at the final concentration $5 \mu \mathrm{M}$ ) was added to the tube and immediately shaked vigorously. Labelling of PBMCs was done promptly 5 minutes at room temperature in the dark. Cells were washed three times in PBS supplemented with 5\% FCS. Thereafter, cRPMI media was added and cells were transferred to cell culture incubator $\left(37^{\circ} \mathrm{C}\right.$ under $\left.5 \% \mathrm{CO}_{2}\right)$ for further experiments.

\section{Flow cytometry and cell sorting}

For the expression of surface markers, cells were stained with the following antibodies: CD3-FITC, CD16-FITC, CD19- ECD, CD19- PC5, CD4-PE/Cy7, CD14-APC/Cy7, CD19-Brilliant Violet 510, CD19APC/Cy7. Matching isotype controls were used as negative controls. Samples were measured with a FACSAria II instrument (Beckton Dickinson) or Galios flow cytometer and analyzed using Kaluza 
software (Beckman Coulter). Dead cell were excluded based on staining with the eFluor 450 dye. Before imaging flow cytometry CD14+ cells were sorted out from rest of the cells accordingly to the CD14-APC/Cy7 signal. To get purified CD14+ cells we excluded from this cell population eFluor 450 positive cells as dead cell population.

\section{Detection of infectious virions}

PBMCs were infected with unlabelled-HRV16 at MOI 10 and incubated for $24 \mathrm{~h}$ at $37^{\circ} \mathrm{C}$ in tissue culture incubator using regular cell culture conditions. Then the PBMCs were washed three times with pre-warmed PBS and added to HeLa cells at a number of 800000 cells per well in a 48-well plate (500 ul volume). Uninfected HeLa cells were seeded in these wells $24 \mathrm{~h}$ before and were growing in log-phase when PBMCs were added. In the ICAM-1-blocking condition, anti-ICAM1-Ab was added to HeLa cells at the concentration of $1 \mathrm{ug} / \mathrm{ml} 2 \mathrm{~h}$ before PBMCs were added. Three days after the addition of PBMCs, HeLa cells were washed thoroughly to remove non-adherent PBMCs and RNA was isolated from HeLa cells using the RNAeasy Micro Plus kit (Qiagen). The expression of negative and positive strands of viral RNA was analyzed by RT-qPCR.

\section{Labelling the virus with DyLight650}

Labelling of viruses with DyLight650 was performed according to manufacturer - Thermo Scientific protocol. Shortly - media component in the virus (both active or UV inactivated) solution was replaced with PBS dialyzing it 2 times for 4 hours with cold PBS (at $\mathrm{pH} 7.0$ and $4^{\circ} \mathrm{C}$ ). For dialyzes, membrane MWCO of 6-8 kDa Spectra/Por $\stackrel{\oplus}{-}$ Dry Standard RC Dialysis Tubing (Spectrumlabs) was used. To the $15 \mathrm{~mL}$ samples was added $1 \mathrm{mg}$ DyLight650 NHS Ester, mixed and stained at RT for 1 hour protected from light. Labelled and starting non-labelled virus solution were titrated by endpoint titration assay in parallel and stored at $-80^{\circ} \mathrm{C}$. Labelling caused the drop of virus infectivity 5 times when to compare with the starting non-labelled virus. 
Cells were labelled and promptly measured by cell imaging flow cytometer "ImageStream". To avoid overlapping of the APC/Cy7 signal with the side scatter signal on the "ImageStream", monocytes were sorted out with the cell sorter FACS Aria before taking images on the "ImageStream". Signal strength was compensated between different channels according to the manufacturer protocols. Flow speed was stabilized before collecting 30000 events and 40x magnification was used for taking images. Data were analyzed using "Ideas" software (Amnis).

\section{QuantiGene ViewRNA in-situ hybridization}

ISH was performed according to manufacturer -Affymetrix, Santa Clara, CA - protocol. Shortly PBMCs were added on top of the polylysine coated microscopic slides. HRV or controls were added to the wells. Before cell fixation, media was carefully replaced with PBS. Thereafter, cells were fixed with 4\% paraformaldehyde and washed with PBS and dehydrated with ethanol at increasing concentrations $(50 \%, 70 \%$, and $100 \%)$. Slides were submerged in $100 \%$ ethanol at $-20^{\circ} \mathrm{C}$ for storage. Before further analysis, cells were rehydrated using decreasing concentrations of ethanol $(70 \%$ and 50\%) and washed with PBS. ISH was performed using QuantiGene ViewRNA protocols designed to detect specifically 20 nucleotide long sequences in HRV VRNA - 15 sites are for amplifying the signal and 5 sites are for blocking the nonspecific binding sites. Cells on the slides were permeabilized with Working Detergent Solution (Affymetrix, Santa Clara, CA) and hybridized for 3 hours at $40^{\circ} \mathrm{C}$ with custom-designed QuantiGene ViewRNA probes against positive strand HRV1B and human actin-beta. Unbound probes were flushed out with Wash Buffer (Affymetrix, Santa Clara, CA). The bound probes were amplified through PreAmp (Affymetrix, Santa Clara, CA) hybridization for 1 hour at $40^{\circ} \mathrm{C}$, followed by Amp (Affymetrix, Santa Clara, CA) hybridization for 1 hour at $40^{\circ} \mathrm{C}$. Label Probes (Affymetrix, Santa Clara, CA) targeting the individual probe types were added for 1 hour at $40^{\circ} \mathrm{C}$. The 
slides were covered in 4',6-diamidino-2-phenylindole (DAPI)-containing mounting medium and examined using an LSM 510 confocal microscope (Zeiss).

\section{Immunofluorescence}

Microscope slides with PBMC after ISH were further incubated 30 minutes with anti-human CD20 (MS4A1 RabMAb ${ }^{\circledR}$, rabbit anti-human, Epitomics 1632-1) or with anti-rabbit polyclonal antibody (IC control), incubated 30 minutes at RT in dark with secondary antibody (Alexa Fluor ${ }^{\circledR} 488$ Goat AntiRabbit IgG $(\mathrm{H}+\mathrm{L})($ Invitrogen, A11034). The slides were covered in DAPI-containing mounting medium and examined using "Leica TCS SPE confocal microscope" (Leica Microsystems).

\section{Cytokine measurement}

PBMCs were cultured in CRPMI with or without HRV ("mock"). After incubation for 5 days, the cells were pelleted, and supernatants were frozen $\left(-80^{\circ} \mathrm{C}\right)$. Cytokines were measured with MILLIPLEX ${ }^{\circledR}$ customized panel of following cytokines - TNF- $\alpha$, IL-7, IL-27, IL-1 $\beta$, IL-9, IL-28, IL-6, IL-10, IL-12p70, IL13, IL-8, IL-17a, RANTES, IFN- $\gamma$, MIP-1 $\alpha$, IP-10, MIP-1 $\beta$, IL-17e/IL-25, IFN- $\alpha 2$, IL-23, IL-5, IL-33. Fluorescent signals were read and analyzed using the Bio-Plex 200 System (Bio-Rad Laboratories).

\section{Statistical analysis}

Unless otherwise indicated, data show mean \pm confidential interval (Cl) $95 \%$. Statistical tests were performed using GraphPad Prism 5.0 and ggplot2. Statistical significance was determined using the 2tailed Student's t test or one-way ANOVA with Bonferroni's Multiple Comparison Test to pinpoint the difference between two groups in multiple selections. Statistical significance was retained for P-value $<0.05$. The number of donors indicated in the figure legends includes all donors analyzed in the relevant experiment. 
While most of the studies in the HRV field have focused on the relationship between HRVs and airway epithelial cells, we were interested in PBMCs; investigating how HRVs and human mononuclear cells interact. First, we cultured PBMCs from healthy donors with different concentrations of HRV1B and determined that HRV1B is able to induce the proliferation of PBMCS at a concentration range $\mathrm{MOI}=1.0 \div 10$ in vitro. At lower and higher viral concentration the proliferation was not detectable or not significantly different from baseline - "mock" - condition (Fig. 1 A). Next we studied, which cell type(s) proliferate in response to HRVs. To measure cell proliferation, we added carboxy-fluorescein succinimidyl ester (CFSE) to the cells on day 0 and cultured PBMCs with HRV1B, HRV14, HRV16 or HRV29 at MOI $=1.0$ or MOI $=10$ for 3 and 5 days, and then stained the CFSE labeled cells with antibodies recognizing CD19, CD4 and CD8 and performed flow cytometry. On the 5 th day after infection, $6-13 \%$ or $14-33 \%$ of CD19+ B cells were detected as proliferating when PBMCs were infected with HRV1B, HRV14, HRV16 or HRV29 at $\mathrm{MOI}=1.0$ or $\mathrm{MOI}=10$, respectively. The remarkable exception was HRV16: using higher viral concentration $\mathrm{MOI}=10$ caused less proliferation of B lymphocytes even when compared with "mock" induced background proliferation. Only a small fraction of CD4+ or CD8+ T cells proliferated with statistically non-significant difference when compared to the "mock" (Fig. 1 B). The proliferation of B cells was noticeable on day 3 , but the difference from "mock" condition was statistically insignificant on day 5 (Fig. 1 C).

To confirm that HRV induced B lymphocyte proliferation is not caused by unspecific stimulation of immune responses caused by the presence of virus components in the medium, we inactivated virus with ultraviolet (UV) radiation. No significant proliferation of PBMCs was observed when UV-treated 
HRV1B in comparison with non-treated HRV1B and HRV16 at MOI 1 and MOI 10 (Fig. 1 D). ICAM-1 blocking partially decreased the proliferation of PBMCs app \%40 (\%14-\%80) during virus stimulation (Fig. $1 \mathrm{E}$ ). This suggests that HRV may directly bind to PBMCs and induce B cell proliferation.

These data suggest HRVs are able to induce B cell proliferation at certain viral concentration and timepoint in vitro.

\section{HRVs can attach or enter to monocytes, CD4+ and CD8+ T cells and B cells}

Next we studied the HRV ability to attach and enter to the lymphocytes as the first events needed for the infection. We labelled HRV1B and HRV16 with fluorescent dye DyLight650 and performed flow cytometry analysis at different timepoints between $30 \mathrm{~min}$ to 24 hours to estimate whether different cell populations take up HRVs. As controls, we used either UV-inactivated HRV1B and HRV16 or "mock" condition. Fig. 2 A and B demonstrate that HRV16 and HRV1B attached and probably also entered into the monocytes already 30 minutes and to $C D 4+T$ and $B$ lymphocytes 8 hours after the addition of the virus. Monocytes were almost $100 \%$ positive for both UV-treated and untreated HRV16 and HRV1B at 24h point of time. This indicates that in monocytes the virus was at least partially taken up via passive internalization and not an active infection. Remarkably, $3.3 \%( \pm 0.2 \%)$ of CD4+ T cells and $2.9 \%( \pm 0.4 \%)$ B cells at 8 h and $14.0 \%( \pm 1.5 \%)$ of CD4+ T cells, $5.9 \%( \pm 1.5 \%)$ of B cells and $7.4 \%( \pm 2.3 \%)$ of $C D 8+T$ cells at $24 \mathrm{~h}$ timepoint were detected to be positive for HRV1B and HRV16 when the higher virus concentration was used. No positive signal of DyLight650 labeled HRV was detected in B cells, CD4+ T cells and CD8+ T-cell at lower virus concentrations and at higher concentration of UV-inactivated virus (Fig. $2 \mathrm{~A}$ and B).

To further characterize the interactions of HRVs with different PBMC subsets, we performed imaging flow cytometry. PBMCs were cultured with higher concentration $(\mathrm{MOI}=10)$ of DyLight650 labelled HRV1B and analyzed at different time-points chosen according to the flow cytometry analysis results. 
Monocytes were analyzed $1 \mathrm{~h}$, while $B$ cells and CD3+/CD4+ and CD3+/CD4- $T$ cells $24 \mathrm{~h}$ after the infection. Consistent with the flow cytometry results, monocytes were detected to be positive for HRV1B $1 \mathrm{~h}$ after the infection and CD4+ T cells and CD19+ B cells 24h after infection (Fig. 2 C, S1-3 Fig.). CD3+/CD4- $T$ cells, representing CD8+ cytotoxic $T$ cell subset, did not give a positive signal in imaging flow cytometry (data not shown). In CD4+ T cells, DyLight650 labelled HRV1B was seen on cell surface where it co-localized with anti-CD4 staining (Fig. 2C, S2 Fig.), while in monocytes and CD19+ B cells, DyLight650 staining pattern indicates that DyLight650 labelled HRV1B was located inside the cells (Fig. 2 C, S1 and S3 Fig.).

\section{HRV forms viral replication centers in B cells and infectious virions in PBMCs}

Next, we used in situ hybridization (ISH) for HRV1B to study whether we are capable of detecting viral RNA (vRNA) that would indicate that HRV could uncoat and infect PBMCs. PBMCs were cultured with infectious HRV1B and ISH was performed at $5^{\text {th }}$ day (Fig. 3 A, S4 Fig.) and as positive control, HeLa cells, were cultured with infectious or UV-treated HRV1B and ISH was performed 1 day after infection (Fig. 3 B). As an additional positive control, we used ISH probes recognizing beta-actin mRNA that is strongly expressed during the activation or division of the cells. In PBMCs, vRNA signal was detected as an intracellular vesicle-like formation with the size around $200-500 \mathrm{~nm}$, which is 10 - 25 times the size of the single viral particle, suggesting that we were able to detect viral replication centers, but not single vRNA with ISH (Fig. 3 A, S4 Fig.). Similar vRNA signal pattern was detected in 4 different donors. More intensive dotted intracellular signal was seen in HeLa cells (Fig. 4 B, S6 Video). Moreover, immunofluorescence analysis demonstrated that the vRNA signal often localized in the cells that stained positively with anti-CD20, a marker for non-terminally differentiated B cells. In conclusion, these data demonstrate that HRV1B can enter into B cells in vitro in cultured PBMCs. To demonstrate that HRV can replicate in PBMCs, we pre-infected PBMCs with HRV16 and seeded them over uninfected HeLa cells after washing then HRV RNA was analysed in stringently washed HeLa 
cells after $72 \mathrm{~h}$. Our data demonstrate that HRV can replicate efficiently in PBMCs and that newly produced virions may have the capacity to infect HeLa cells, as demonstrated by measuring viral RNA in the HELA cells. Figure $3 \mathrm{C}$ shows the expression of positive and negative strand viral RNAs in HeLa cells after all of the above procedures. UV irradiated HRV was not able to infect PBMCs and therefore virus was not able to replicate and reinfect HELA cells. Blocking of ICAM-1 on HeLa cells was leading to a reduced infection rate in HELA cells to about \%57 (\%24-\%84) of the virus load in average that was observed for the non-blocked infection condition. The same results were obtained in both positive and negative strand mRNA detections.

\section{The cytokine profile of HRV-infected PBMCs}

To explore whether and how different HRVs stimulate immune responses that possibly support the observed B cell proliferation, we next determined the profile of chemokines/cytokines produced by PBMCs. We infected cultured PBMCs using the same experimental settings as in Fig. 2-5, collected the supernatants on day 5 after HRV infection and measured the panel of 27 cytokines. All used virus strains upregulated IL-6, IFN- $\alpha$, IL-10, IFN- $\gamma$ and RANTES. MIP1 $\beta$, IL-1beta, IL-6, and TNF- $\alpha$ were stimulated more strongly upon HRV16 infection (Fig. 4). All viruses induced IFN- $\alpha$ production, but minor group rhinoviruses, HRV1B and HRV29, induced significantly stronger expression of IFN- $\alpha$ at MOI=10. For major group viruses, HRV16 and HRV14, there was no significant concentration dependent difference in the induction of IFN- $\alpha$. IFN- $\gamma$ was significantly stronger expressed at all virus types compared to "mock" with the exception HRV16 MOI=10 and HRV29 MOI=1, where there was no significant difference. Interestingly, HRV16 MOI=10 concentration did not increase the IFN- $\gamma$ production and at the same time IL-10 production was strongly upregulated. IL-27 was upregulated by major HRV types, HRV16 and HRV14 and was observed to be more strongly expressed at MOI=1. These results show that HRVs induce immune responses in cultured PBMCs with moderate differences between the virus strains. 
The mechanisms of the development of immune responses to HRVs and the causal relationship between HRV infection and asthma exacerbation are not completely understood. In the current study, we demonstrate that monocytes, CD4+ T cells, CD19+ B cells, and CD8+ T cells are able to interact with HRVs in in vitro cultured PBMCs. More importantly, we show that HRVs enter and can induce the proliferation of CD19+ B-cells. In addition, we demonstrate that HRVs activate PBMCs and induce the production of inflammatory cytokines MIP-1 $\beta$, IL-1 $\beta$, IL-6, TNF- $\alpha$, IFN- $\alpha$, IL-10, RANTES, IFN- $\gamma$ and IL-27.

During the last several decades, strong evidence have accumulated supporting the idea that HRV infections enhance allergic inflammation in the airway leading often to increased asthmatic symptom - asthma exacerbation (2, 21-23). Most commonly, HRVs infect only URT epithelial cell lining, and for most of us, HRV infection causes only a relatively mild illness with upper respiratory symptoms as seen during the common cold (24). Remarkably, more than $50 \%$ of bronchial epithelial biopsies collected from patients during virus induced asthma exacerbation have found to be HRV positive (25).

While the research has been focused mostly on viral infection in bronchial epithelia as the principal site of viral replication, the capacity of HRVs to directly infect immune cells and to modulate immune responses has been studied less. Still, Levandowski et al. demonstrated that the total lymphocyte count of infected individuals was significantly reduced on day 3 after the challenge with a rhinovirus of the minor group(26). In addition, they showed that specifically T lymphocyte numbers were reduced, while B lymphocyte levels were constant. Decreased cell populations positively correlated with disease severity. In addition, virus shedding was the highest in individuals with the greatest reduction in lymphocytes. The reduced number of lymphocytes in the circulation might be at least partly explained by a massive lymphocytic and eosinophilic infiltration into the infected tissues. This 
cell infiltration to bronchial mucosa might be responsible for changes in airway hyper-responsiveness and asthma exacerbations(27). Viremia may occur during HRV respiratory infections, suggesting that rhinoviremia may be involved in asthma exacerbation pathogenesis(28). In addition, Gern et al. showed that the activation of T cells estimated by CD69 was detectable at HRV16 MOI of 0.03, with maximum CD69 expression occurring with a MOI of 1 (29). UV-irradiation did not significantly increase T cell CD69 expression. The narrow range of viral concentration was similar to what was observed in our experiments with B cells. B cell proliferation reached its highest values on days 3 to 5 while the HRV induces no statistically significant proliferation of B lymphocytes during first $48 \mathrm{~h}$ as it has been noticed already by Papadopoulos et al. but still they observed a suboptimal Th1 response in PBMC from atopic asthmatic subjects and differential expression of costimulatory molecules(30). It was also dependent on the virus strain and concentration, which was optimum around $\mathrm{MOI} 1$ and 10 showing difference between the viral strains.

As a novel approach, we followed the dynamics of virus attachment and uptake by different PBMC populations using virus particles labelled with DyLight650. Uptake of the HRV by all leukocytes was observed 20 years ago using $\left[{ }^{35} \mathrm{~S}\right] \mathrm{HRV} 16$ and from these experiments was known that HRV16 attaches to all type of leukocytes and has fourfold higher binding effect to monocytes (29). We directly visualized the attachment by imaging flow cytometry and describe here first time the kinetics of it by conventional flow cytometry. DyLight650 dye contains N-hydroxysuccinimide (NHS) esters, what react with the amines on the virus capsid proteins and thereby labelling the viral capsid proteins with red fluorescent dye. One should understand that labelling is present only on the parent generation of viruses and it will be lost during viral replication. We observed that monocytes internalize HRV and UV-inactivated viruses. This indicates that the uptake of HRV particles by monocytes is at least partially a passive event and not only via receptor mediated infection. B and T lymphocytes had distinct attachment and/or internalization pattern in case of functional virus and no signal with UV-treated viruses detected. There was a lag time around 8h before HRV was detected in 
B and CD4+/CD8+ T cells. In case of CD4 T cells, the DyLight650 labelled virus signal overlapped CD4 signal that points to the attachment of virus to CD4. Our results were concordant with the previously reported results about attachment of HRV to different types of leukocytes(29) but give better understanding of the kinetics of the process what allows better to plan similar experiments to further study HRV leukocytes interactions.

In addition to DyLight650 labelling, we used ISH for viral RNA as the second visualization method. We were able to detect accumulation of vRNA on the surface and inside of B cells. The size of the vRNA signal, which is found on B cells indicates that these clusters are viral replication centers. Additionally we demonstrate that HRV infected PBMCs were able to transfer the infectious particles to other cells, as shown by measuring virus RNA-expression in HELA cells after co-culture with pre-infected PBMCs. These finding indicate strongly that HRV is able to replicate inside the peripheral blood mononuclear cells. It might seem to contradict with the study conducted 20 years ago where it was shown that the viral titers decrease over time when PBMCs were exposed to HRV16 (MOI = 10, 72h) suggesting that no type of cells in PBMCs develops effective infection(29). Still the authors found HRV RNA in the cytoplasm of monocytes as we did, but no viral RNA synthesis was detected. But other studies showed later that under certain conditions (especially timing and choice of "right" viral concentration are important) HRV is still able to infect alveolar macrophages(12), T cells(13) and Ramos cells (B cell line)(16). Definitely the discussion stays open while the studies are conducted in vitro and the topic needs further studies to clarify if the infection of lymphocytes (beside alveolar macrophages) is relevant in vivo.

We observed that HRVs are able to induce B-cell proliferation. The rate of proliferation was time, virus type and dose dependent. HRV-induced lymphocyte proliferation was slightly detectable at lower $(\mathrm{MOI}<1.0)$ viral concentrations, heavily dampened at higher concentrations $(\mathrm{MOI}>10)$ and clearly visible in the range of $\mathrm{MOI}=1.0 \div 10$. HRV16 was exceptional as the proliferation dampening 
happened earlier at lower viral concentration $(\mathrm{MOI}=10)$ suggesting that its virulence might be stronger. Similar to our results with HRVs, other picornaviruses, such as poliovirus and coxsackievirus efficiently induce proliferation of B cells, which start to produce IgM independently from T-cell help (31). As it is known that T cell independent antibody response results from extensive B-cell receptor cross-linking by the highly organized, repetitive picornavirus virion structure and is postulated to be generally characteristic of antibody-controlled cytolytic viruses (31).

It has been shown that major group rhinoviruses significantly inhibit T-cell proliferation $(32,33)$. We did not observe induction of neither CD4+ nor CD8+ T-cell proliferation in PBMCs with any of used virus strains even in the conditions where B lymphocytes proliferated. T cell proliferation upon HRV was confirmed couple of years ago, but as authors pointed out the viral concentration needed to be very low -1000 more diluted - and the T cell proliferation was detected later on the day $5-7$ when compare with our experiments and it explains why we did not detect the T cell proliferation, but these two studies show how sensitive is the event of lymphocyte proliferation to the chosen condition. The mechanism how HRV inhibits antigen-specific CD4+ or CD8+ T cell proliferation is known to be ICAM-1-dependent (32). Our results indicate that there might be a similar regulatory mechanism through the LDL receptor as minor group HRVs, HRV1B and HRV29, also did not induce Tcell proliferation.

A recent study demonstrated that B-lymphocytes play an important role in the induction of asthmalike inflammation in mice (34). It has been also suggested that B-lymphocytes may become increasingly relevant as antigen-presenting cells when antigen load is low even without the help of CD4+ T-lymphocytes (35). It is possible that in allergic/asthmatic individuals, HRVs infect not only LRT epithelial cells but also underlining infiltrating immune cells in the inflamed epithelium, which ultimately may lead to a strong nonspecific induction of B cell proliferation in bronchial tissue. This 
might lead to a rapid increase in the total serum IgE, as observed previously (36) and thereby can provide synergistic link between allergy, HRV infection and exacerbation of asthma (20).

In conclusion, this study provides first time evidence that HRVs can be taken up and induce the proliferation of B lymphocytes and suggests that the mechanism how HRV induces B lymphocyte proliferation and activation can be promising target for new therapeutic intervention strategies for asthma exacerbation.

\section{Figures}

Figure 1 The capacity of HRV to induce proliferation of PBMCs and B cells. (A) Proliferation rate was measured by using ${ }^{3} \mathrm{H}$-thymidine incorporation assay. PBMCs were incubated with HRV1B at indicated concentrations for 5 days. Stimulation index is a ratio of counts per minute (CPM) in virus induced versus "mock" induced condition. Data are mean (line) with Cl 95\% (grey area) from 11 different donors. (B, C, D) Proliferation rate of $B$ cells and $C D 8+$ and $C D 4+$ cells was measured using CFSE labelling assay and anti-CD4, anti-CD8 and anti-CD19 antibodies as discrimination markers. PBMCs were incubated with mock, HRV1B, HRV16 (MOI=1.0 or 10.0) and with UV inactivated HRV1B or HRV16 for 3 and/or 5 days. Number of samples was 6 . Data are mean with ${ }^{* *} \mathrm{P}<0.01,{ }^{*} \mathrm{P}=$ $0.01 \div 0.05$ from 6 different donors. (E) Addition of anti-ICAM-1-antibody to PBMCs leads to a marked reduction in PBMC proliferation compared to isotype control. PBMCs were infected with $\mathrm{MOI} 10$ of HRV16. Number of samples was 4.

Figure 2 Analysis of PBMCs incubated with DyLight650 labelled HRV1B and HRV16. PBMCs were cultured with DyLight650 labelled HRV1B or HRV16 at MOI = 1.0 and MOI = 10 or "mock" and analyzed at the indicated time-points by flow cytometry (A, B) or imaging flow cytometry (C). The UV treated viruses were used at $\mathrm{MOI}=10 .(\mathrm{A}) \mathrm{A}$ representative of the flow cytometry. (B) Data are mean with $\mathrm{Cl} 95 \%$ of 6 donors. (C) Imaging of HRV interactions with monocytes, T and B cells. 
Brightfield (BF) image shows the shape of cells. CD14+ monocytes were sorted out $1 \mathrm{~h}$ after incubation with the virus by cell sorter and then analyzed for DyLight650-labelled HRV1B (red). T lymphocytes were analyzed for surface markers using anti-CD3 FITC (green) and anti-CD4 PE/Cy7 (purple) and for DyLight650-labelled HRV1B (red) 24h after incubation with the virus. B lymphocytes were analyzed with anti-CD19 FITC (green) for DyLight650-labelled HRV1B (red) after 24h incubation with the virus.

Figure 3 Visualization of HRV1B vRNA with in situ hybridization (ISH). HRV1B VRNA is designated with green, human beta-actin mRNA with red, DAPI with blue, and anti-CD20 (purple arrow) as the marker for B-cells with purple color. (A) PBMCs were cultured with HRV1B and subjected to ISH analysis on $5^{\text {th }}$ day. Representative images of 4 donors on $5^{\text {th }}$ day are presented. White arrows indicate the vRNA signal. Bars indicate $10 \mu \mathrm{m}$. (B) HeLa cells were infected with HRV1B or HRV1B UV. After 24 hours cells were fixed and subjected to ISH analysis. Bars indicate $10 \mu \mathrm{m}$. (C) HRV can successfully replicate in PBMCs and produce infectious virions, which are able to infect HELA cells. Expression of positive strand and negative strand viral RNA in HELA cells after co-culture with pre HRV incubated PBMCs for three days are shown.

Figure 4 HRV-induced cytokine responses of PBMCs. PBMCs were incubated with HRV1B, HRV16, $\mathrm{HRV} 14$ and $\mathrm{HRV} 29$ at $\mathrm{MOI}=1.0$ and $\mathrm{MOI}=10$ concentrations or cultured in "mock" condition. Cell culture supernatants were collected at day 5 . Data are mean with $\mathrm{Cl} 95 \%$ of 8 donors. ${ }^{* *} \mathrm{P}<0.01, * \mathrm{P}=$ $0.01 \div 0.05$

\section{Author contributions}

AA had primary responsibility for framework of the study, outcome assessment and manuscript preparation. OW produced substantial amount of data during revising the manuscript and deserves to share first author ship together with AA. AA, WV, AR, ME, SJ and CA contributed to the conception 
and design of the study, and MA supervised the project. AA, OW, SS, BS, BR and JA performed the experiments. AA, OW, WV, BS, AR, CA and MA conducted data analysis and interpretation of the results. NGP provided help for the planning of additional experiments and interpretation of the data during revising the manuscript. All authors contributed to revision of the manuscript.

\section{Funding}

M. Akdis has received research support from the Swiss National Science Foundation No. 320030159870 and the European Commission's Seventh Framework Programme MeDALL (No: 261357) and PREDICTA (No: 260895). C.A. Akdis has consultant arrangements with Actellion, Aventis, Stallergenes, Allergopharma, and Circacia; is employed by the Swiss Institute of Allergy and Asthma Research, University of Zurich; and has received research support from Novartis, the Swiss National Science Foundation No. 310030_156823, and the Christine Kühne-Center for Allergy Research and Education (CK-CARE). A. Rebane has received research support from European Regional Fund with Archimedes Foundation, EU structural assistance grant SARMP12219T, personal research grant PUT214 from Estonian Research Council and European Union through the European Regional Development Fund (Project No. 2014-2020.4.01.15-0012).

\section{Conflicts of interest}

The authors declare that they have no potential conflict of interest.

\section{Supporting Information}

Figure S1 Imaging of HRV uptake by monocytes. Brightfield (BF) image shows the shape of cells. CD14+ monocytes were sorted out $1 \mathrm{~h}$ after incubation with the virus by cell sorter and then analyzed for DyLight650-labelled HRV1B (red) (A) and DyLight650-labelled and UV-treated HRV1B (B).

Figure S2 Imaging of HRV uptake by CD4+ T-cells. Brightfield (BF) image shows the shape of cells. CD4+ T lymphocytes were analyzed for surface markers using anti-CD3 FITC (green), anti-CD4 PE/Cy7 
477 (purple) and for DyLight650-labelled HRV1B (red) 24h after incubation with the virus. PBMCs were

478 cultured with DyLight650-labeled HRV1B (A) and DyLight650-labelled and UV-treated HRV1B (B) and

479 CD4+ T-cells were sorted out $24 \mathrm{~h}$ after incubation with the virus by cell sorter.

480

481

482

483

484

485

486

487

488

489

490

491

492

493

494

495

496

497

498

499

500

501

502

503

Figure S3 Imaging of HRV uptake by CD19+ B-cells. Brightfield (BF) image shows the shape of cells. B lymphocytes were analyzed for surface markers using anti-CD19 FITC (green) and for DyLight650labelled HRV1B (red) 24h after incubation with the virus. PBMCs were cultured with DyLight650labeled HRV1B (A) and DyLight650-labelled and UV-treated HRV1B (B) and B lymphocytes were sorted out $24 \mathrm{~h}$ after incubation with the virus by cell sorter.

Figure S4 Donor 5 (D5) - HRV1B is inside the B cell. HRV1B vRNA is designated with green, human beta-actin mRNA with red, DAPI with blue, and anti-CD20 as the marker for B-cells with purple color. PBMCs were cultured with HRV1B and subjected to ISH analysis on $5^{\text {th }}$ day. Cells images are different slices to visualize the localization of HRV replication centers.

\section{References}

1. Johnston SL, Pattemore PK, Sanderson G, Smith S, Lampe F, Josephs L, et al. Community study of role of viral infections in exacerbations of asthma in 9-11 year old children. BMJ 1995;310(6989):1225-1229.

2. Nicholson KG, Kent J, Ireland DC. Respiratory viruses and exacerbations of asthma in adults. BMJ 1993;307(6910):982-986.

3. Johnston SL. Overview of virus-induced airway disease. Proc Am Thorac Soc 2005;2(2):150156.

4. McIntyre CL, Knowles NJ, Simmonds P. Proposals for the classification of human rhinovirus species A, B and C into genotypically assigned types. J Gen Virol 2013;94(Pt 8):1791-1806.

5. Jacobs SE, Lamson DM, St George K, Walsh TJ. Human rhinoviruses. Clin Microbiol Rev 2013;26(1):135-162.

6. Bochkov YA, Watters K, Ashraf S, Griggs TF, Devries MK, Jackson DJ, et al. Cadherin-related family member 3 , a childhood asthma susceptibility gene product, mediates rhinovirus $\mathrm{C}$ binding and replication. Proc Natl Acad Sci U S A 2015;112(17):5485-5490. 
7. Bønnelykke K, Sleiman P, Nielsen K, Kreiner-Møller E, Mercader JM, Belgrave D, et al. A genome-wide association study identifies CDHR3 as a susceptibility locus for early childhood asthma with severe exacerbations. Nat Genet 2014;46(1):51-55.

8. Yong K, Khwaja A. Leucocyte cellular adhesion molecules. Blood Rev 1990;4(4):211-225.

9. De Sanctis JB, Blanca I, Rivera H, Bianco NE. Expression of low-density lipoprotein receptors in peripheral blood and tonsil B lymphocytes. Clin Exp Immunol 1998;113(2):206-212.

10. Yanai I, Benjamin H, Shmoish M, Chalifa-Caspi V, Shklar M, Ophir R, et al. Genome-wide midrange transcription profiles reveal expression level relationships in human tissue specification. Bioinformatics 2005;21(5):650-659.

11. Jakiela B, Gielicz A, Plutecka H, Hubalewska-Mazgaj M, Mastalerz L, Bochenek G, et al. Th2type cytokine induced mucous metaplasia decreases susceptibility of human bronchial epithelium to rhinovirus infection. Am J Respir Cell Mol Biol 2014.

12. Laza-Stanca V, Stanciu LA, Message SD, Edwards MR, Gern JE, Johnston SL. Rhinovirus replication in human macrophages induces NF-kappaB-dependent tumor necrosis factor alpha production. J Virol 2006;80(16):8248-8258.

13. Ilarraza R, Wu Y, Skappak CD, Ajamian F, Proud D, Adamko DJ. Rhinovirus has the unique ability to directly activate human T cells in vitro. J Allergy Clin Immunol 2013;131(2):395-404.

14. Stokes CA, Kaur R, Edwards MR, Mondhe M, Robinson D, Prestwich EC, et al. Human rhinovirus-induced inflammatory responses are inhibited by phosphatidylserine containing liposomes. Mucosal Immunol 2016.

15. Graser A, Ekici AB, Sopel N, Melichar VO, Zimmermann T, Papadopoulos NG, et al. Rhinovirus inhibits IL-17A and the downstream immune responses in allergic asthma. Mucosal Immunol 2016.

16. Rager KJ, Langland JO, Jacobs BL, Proud D, Marsh DG, Imani F. Activation of antiviral protein kinase leads to immunoglobulin E class switching in human B cells. J Virol 1998;72(2):1171-1176.

17. Kirchberger $S$, Majdic $O$, Stockl J. Modulation of the immune system by human rhinoviruses. Int Arch Allergy Immunol 2007;142(1):1-10.

18. Gavala ML, Bertics PJ, Gern JE. Rhinoviruses, allergic inflammation, and asthma. Immunological Reviews 2011;242(1):69-90.

19. Gern JE, Rosenthal LA, Sorkness RL, Lemanske RF. Effects of viral respiratory infections on lung development and childhood asthma. J Allergy Clin Immunol 2005;115(4):668-674; quiz 675.

20. Olenec JP, Kim WK, Lee WM, Vang F, Pappas TE, Salazar LE, et al. Weekly monitoring of children with asthma for infections and illness during common cold seasons. J Allergy Clin Immunol 2010;125(5):1001-1006.e1001.

21. Hashimoto S, Matsumoto K, Gon Y, Ichiwata T, Takahashi N, Kobayashi T. Viral infection in asthma. Allergol Int 2008;57(1):21-31. 
22. Rosenthal LA, Avila PC, Heymann PW, Martin RJ, Miller EK, Papadopoulos NG, et al. Viral respiratory tract infections and asthma: the course ahead. J Allergy Clin Immunol 2010;125(6):12121217.

23. Kim WK, Gern JE. Updates in the relationship between human rhinovirus and asthma. Allergy Asthma Immunol Res 2012;4(3):116-121.

24. Bush RK, Busse W, Flaherty D, Warshauer D, Dick EC, Reed CE. Effects of experimental rhinovirus 16 infection on airways and leukocyte function in normal subjects. J Allergy Clin Immunol 1978;61(2):80-87.

25. Wos M, Sanak M, Soja J, Olechnowicz H, Busse WW, Szczeklik A. The presence of rhinovirus in lower airways of patients with bronchial asthma. Am J Respir Crit Care Med 2008;177(10):1082-1089.

26. Levandowski RA, Ou DW, Jackson GG. Acute-phase decrease of T lymphocyte subsets in rhinovirus infection. J Infect Dis 1986;153(4):743-748.

27. Fraenkel DJ, Bardin PG, Sanderson G, Lampe F, Johnston SL, Holgate ST. Lower airways inflammation during rhinovirus colds in normal and in asthmatic subjects. Am J Respir Crit Care Med 1995;151(3 Pt 1):879-886.

28. Xatzipsalti M, Kyrana S, Tsolia M, Psarras S, Bossios A, Laza-Stanca V, et al. Rhinovirus viremia in children with respiratory infections. Am J Respir Crit Care Med 2005;172(8):1037-1040.

29. Gern JE, Vrtis R, Kelly EA, Dick EC, Busse WW. Rhinovirus produces nonspecific activation of lymphocytes through a monocyte-dependent mechanism. J Immunol 1996;157(4):1605-1612.

30. Papadopoulos NG, Stanciu LA, Papi A, Holgate ST, Johnston SL. Rhinovirus-induced alterations on peripheral blood mononuclear cell phenotype and costimulatory molecule expression in normal and atopic asthmatic subjects. Clin Exp Allergy 2002;32(4):537-542.

31. Bachmann MF, Zinkernagel RM. The influence of virus structure on antibody responses and virus serotype formation. Immunol Today 1996;17(12):553-558.

32. Gern JE, Joseph B, Galagan DM, Borcherding WR, Dick EC. Rhinovirus inhibits antigen-specific T cell proliferation through an intercellular adhesion molecule-1-dependent mechanism. $J$ Infect Dis 1996;174(6):1143-1150.

33. Kirchberger S, Majdic O, Steinberger P, Blüml S, Pfistershammer K, Zlabinger G, et al. Human rhinoviruses inhibit the accessory function of dendritic cells by inducing sialoadhesin and B7-H1 expression. J Immunol 2005;175(2):1145-1152.

34. De Vooght V, Carlier V, Devos FC, Haenen S, Verbeken E, Nemery B, et al. B-lymphocytes as Key Players in Chemical-Induced Asthma. PLoS One 2013;8(12):e83228.

35. Lund FE, Randall TD. Effector and regulatory B cells: modulators of CD4+ T cell immunity. Nat Rev Immunol 2010;10(4):236-247. 
573 36. Skoner DP, Doyle WJ, Tanner EP, Kiss J, Fireman P. Effect of rhinovirus 39 (RV-39) infection on 574 immune and inflammatory parameters in allergic and non-allergic subjects. Clin Exp Allergy 575 1995;25(6):561-567. 